\title{
ST Reliability and Connectivity of VANETs for Different Mobility Environments
}

\author{
Hussain Saajid ${ }^{1}$, WU DI ${ }^{1}$, Sheeba Memon ${ }^{2}$ and \\ Naadiya Khuda Bux ${ }^{2}$ \\ ${ }^{1}$ School of computer science and engineering Dalian University of Technology \\ [e-mail: sunny_sau@hotmail.com,wudi@dlut.edu.cn] \\ ${ }^{2}$ School of information science and engineering Central South University \\ Changsha, Hunan, China. \\ [e-mail: memon.sheeba@yahoo.com,naadiya.khudabux@yahoo.com] \\ *Corresponding author: WU DI \\ Received June 8, 2018; revised October 20, 2018; accepted November 5, 2018; \\ published May 31, 2019
}

\begin{abstract}
Vehicular ad-hoc network (VANET) is the name of technology, which uses 'mobile internet' to facilitate communication between vehicles. The aim is to ensure road safety and achieve secure communication. Therefore, the reliability of this type of networks is a serious concern. The reliability of VANET is dependent upon proper communication between vehicles within a given amount of time. Therefore a new formula is introduced, the terms of the new formula correspond 1 by 1 to a class special ST route (SRORT). The new formula terms are much lesser than the Inclusion-Exclusion principle. An algorithm for the Source-to-Terminal reliability was presented, the algorithm produced Source-to-Terminal reliability or computed a Source-to-Terminal reliability expression by calculating a class of special networks of the given network. Since the architecture of this class of networks which need to be computed was comparatively trivial, the performance of the new algorithm was superior to the Inclusion-Exclusion principle. Also, we introduce a mobility metric called universal speed factor (USF) which is the extension of the existing speed factor, that suppose same speed of all vehicles at every time. The USF describes an exact relation between the relative speed of consecutive vehicles and the headway distance. The connectivity of vehicles in different mobile situations is analyzed using USF i.e., slow mobility connectivity, static connectivity, and high mobility connectivity. It is observed that $p_{c}$ probability of connectivity is directly proportional to the mean speed $\mu_{v}$ till specified threshold $\mu_{\tau}$, and decreases after $\mu_{\tau}$. Finally, the congested network is connected strongly as compared to the sparse network as shown in the simulation results.
\end{abstract}

Keywords: Reliability, expression of reliability, I.E principle; vehicular ad-hoc networks, headway distance, freeway highway, universal speed factor. 


\section{Introduction}

Several studies have been conducted to examine the reliability of networks in terms of connectivity, according to the network topology [1] [2]. These approaches have been categorized into three wide classes: Pivotal Decomposition (PD), Inclusion-Exclusion (IE) and Sum of Disjoint Products (SDP) [3] [4]. The reliability of networks is gaining importance day by day particularly safety-sensitive networks like Vehicular ad hoc Networks. A probability graph model was proposed by Colbourn and AboEIFotoh, several special wireless networks have been studied, also presented an estimated algorithm [5].

In networks, Source-to-Terminal reliability refers to the probability that there prevail no less than one possible connection amongst two vehicles under the specified condition. Reliability issues appear increasingly essential as advance networks turn to be increasingly unpredictable. This encouraged the research on the reliability of networks, which drew a lot of attention during previous decades [6] [7]. The term connectivity refers to measure the performance of vehicular communication on superhighways. With the integration of 5G, an exponential growth in VANETs technology is observed [8] [9]. When VANETs and IoT (internet of things) are combined that is called IoV [8]. When vehicles are combined with internet, sensors and road equipments, VANETs comes with multiple insights [10]. The important research issue in VANETs is communication. By using V2V and V2R the goal of VANETs is to present safety and non-safety applications. On the highways to keep vehicles strongly connected with each other multi-hop ad-hoc connections are used. The efficiency of VANETs connectivity depends on the reliable and robust connection.

The research of connectivity deters to another track by introducing VANETs. An exponential increase is observed in the field of network connectivity analysis and that's the reason of attraction for various researchers [11] [12] [29] [30] [31][36]. On the highway, if two vehicles belong to the transmission range of each other, they are said to be connected. To ensure reliable and real-time data distribution, the network should be strongly connected [13]. The impacts of vehicles connectivity in various scenarios have been discussed by many researchers [13] [14] [15] [27] [28]. The extended version of conventional cloud technology is Vehicular Cloud Computing (VCC), which enhances the advantages of VANET [16].

Based on Ant Colony Optimization (ACO) a novel Clustering algorithm is proposed for VANET called ACONET. In order to achieve robust communication for VANETs the algorithm forms optimized clusters. For optimized clustering, parameters of transmission range, load balance factor (LBF) and speed of the nodes are considered [32].

A two-stage approach (SubBus), is introduced which is made-up of dynamic routes planning and travel requirement prediction, based on several crowdsourced shared bus data to produce dynamic routes for shared buses in the "last mile" scene [33]. Based on regional function discovery using several mobile data a co-occurrence pattern mining scheme (CoPFun) is presented [34].

To rescale heterogeneous traffic trajectory data an integrated computing method is presented, which leverages MLE and BIC [35]. The relationship between the mobility and connectivity of VANETs is studied. To achieve this goal, some key topology metrics are employed, including component size and component speed, to describe mobility and connectivity [25]. 
In VANETs, mostly the models based on connectivity simply assume that each vehicle has same speed at every time or either not same as others, but similar for a specified vehicle throughout the entire journey [17] [14] [18] [19]. The assumption of the same velocity will further degrade the performance of the distribution model for inter-vehicle space significantly. To measure the mobility scenario of two connected vehicles relative speed is a good parameter. No matter whether $v_{i}$ and $v_{j}$ (vehicles) are moving very fast, but their connectivity will not be affected, because of their smaller relative speed.

To measure the relationship between vehicles speed and inter-vehicle space a mobility metric named speed factor was proposed by the authors of [14]. They have considered the same speed at all time for every vehicle. The relationship between arrival rate and speed was ignored. Usually, speed varies on the driving behavior and rate of arrival to the road segment. Assume at all time, keeping the constant speed to a typical traffic situation, then the headway distance cannot be completely independent and identically distributed (i.i.d) also the distribution cannot be exponential [13] [14] [15].

A new protocol named MAC connectivity-aware for platoon structure was proposed by the authors in [20] [15]. In their model, they have considered connectivity as a function of node density and its affects was examined in different highway scenarios. According to the results obtained by the authors of [20], it was clear that the increase in connectivity up-to some level will increase throughput, but also throughput decreases after that level. The ideas and methodology offered by [13], is followed by the authors of [20] and [15] in their models, but vehicular platoons were focused.

This paper introduces a new formula for ST reliability of VANETs the formula holds terms which correspond 1 by 1 to a class special ST route (SRORT). For a specified network, the enclosed terms of the formula are less than the Inclusion-Exclusion principle. An algorithm which generates Source-to-Terminal reliability expression of is presented. The algorithm computes the Source-to-Terminal reliability or creates a Source-to-Terminal reliability expression by counting a class of special networks of the given network. The reliability expression obtained by this algorithm is shorter than the Inclusion-Exclusion principle.

We also proposed a mobility metric named universal speed factor (USF) by utilizing the relative speed of associated vehicles. The proposed USF describes an exact relation between relative speed and connectivity of vehicles. Three different scenarios are considered to analyze the performance of USF that is slow mobility connectivity [20-40] Km/h, static connectivity, and high mobility. As compared to the sparse network, the congested network will be more connected as shown in the simulation results.

The rest of the paper is organized as follows, next Section defines definitions used in this paper. Section III describes the new topological formula and Algorithm in detail, IV explains universal speed factor in detail, Section V gives connectivity of VANETs in various mobility environments, Section VI presents obtained results from simulations with discussion and VII conclusion respectively. 


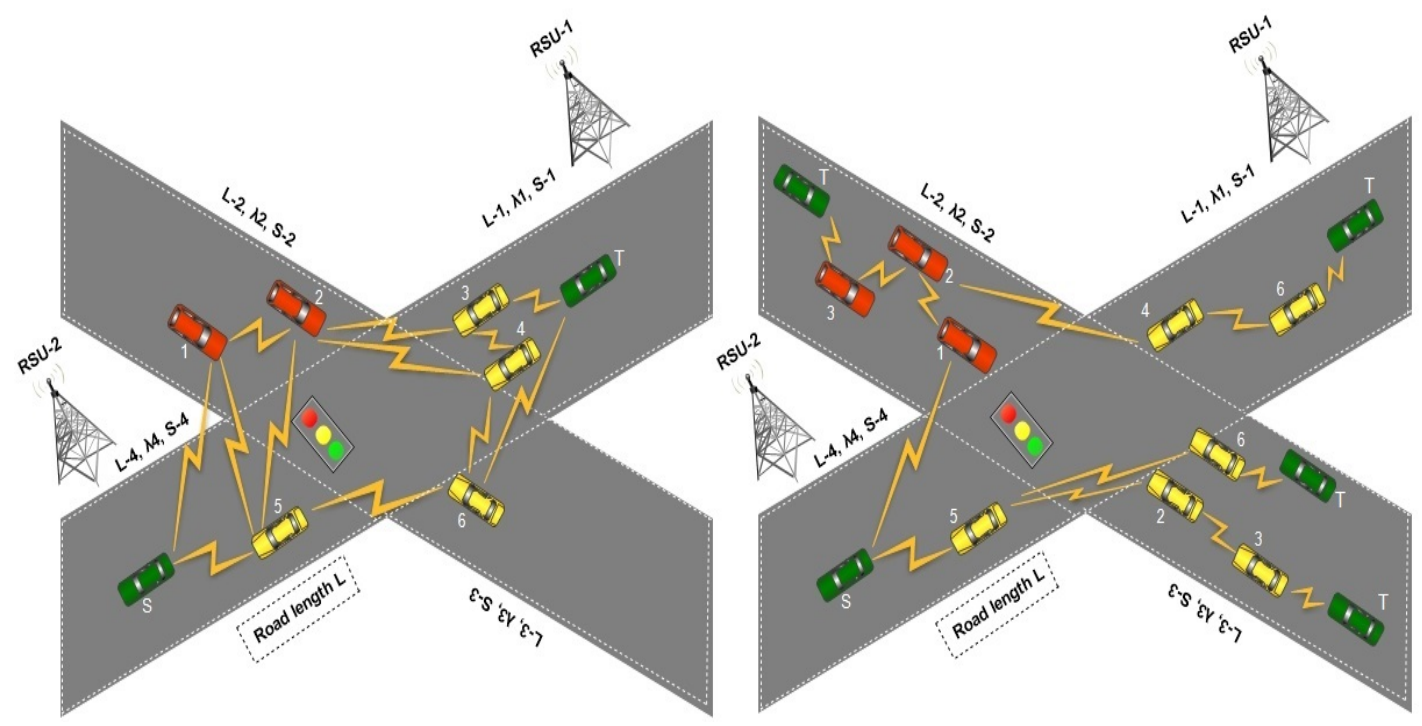

Fig. 1. $\left(\boldsymbol{V}_{\boldsymbol{T}}\right)$ VANET and its SRORT tree with universal speed factor model

\section{Notations \& Definitions}

\subsection{Definitions}

$R O_{i j}$ : A route from i to $\mathrm{j}$.

$S R O_{i j}$ : A route from i to $\mathrm{j}$, and the set of vehicles on the route has not the subset that can create $S R O_{i j}$ we name it as simplest route $S R O_{i j}$.

SRORT: $\mathrm{S}$ is the root vehicle of the tree, $\mathrm{T}$ is the leaf vehicles, middle vehicles are the other vehicles of $V_{T}$, each route from Source-to-Terminal in the tree corresponding 1 by 1 the $S R O_{i j}$ of $V_{T}$.

$V_{T}(V, X)$ : VANET, $\mathrm{V}$ is the set of the vehicles of $V_{T}$, $\mathrm{X}$ is the set of the links of $V_{T}$.

$S, T$ : The Source and the Terminal vehicles of $V_{T}$.

$m$ : The number of $\operatorname{SRO}(S, T)$ of $V_{T}$.

$S_{i}$ : The set of the sons of vehicle i.

$K_{R O}$ : The set of the vehicles in route $\mathrm{RO}$.

$y_{i}$ : A vehicle in the $S R O R T$.

$R\left(V_{T}\right)$ : Source-to-Terminal reliability of vehicular ad hoc networks. 


\section{Reliability of VANET: The New Topological Formula and Rapid Algorithm}

We consider a vehicular ad-hoc networks $\left(V_{T}\right) \quad V_{T}(V, X), V=(1,2,3, \ldots n), X=$ $x_{1}, x_{2}, x_{3}, \ldots x_{x}, n=|V|, x=|X|$. The probability that vehicle $\mathrm{i}$ is working is $p_{i}$, where the failure probability is $1-p_{i}$.

The definition of the Reliability of vehicular ad hoc networks [21]:

$R\left(V_{T}\right)=$ the probability of having a $R O(S, T)$ in $V_{T}$ that each vehicle in the $R O(S, T)$ is working.

$$
R\left(V_{T}\right)=\operatorname{pr}\left(\sum_{i=1}^{n} p_{i}\right)
$$

According to the algebraic axiom $P(a+a c)=P(a)$, if $R O(S, T)$ is not the simple route $S R O(S, T)$, then $K_{R O}$ (the vehicles set of $R O(S, T)$ ) has a subset $K_{S R O}$, that can build up a $S R O$ $(S, T), K_{S R O} \subset K_{R O}, P(R O+S R O)=P(S R O)$ therefore:

Theorem1. $R\left(V_{T}\right)=P\left(\sum_{i=1}^{m} S R O_{i}\right), i=1,2,3, \ldots m$, the number of $S R O(S, T)$ of $V_{T}$ is $m$.

Theorem2. If $S R O$ is a Source-to-Terminal simple route, then it can only be confirmed by its vehicle set $K_{S R O}$.

Prove: The range of $S R O$ is $|S R O|=\left|K_{S R O}\right|-1$, assume $S R O=S y_{1} y_{2} \ldots y_{k-1} T$, since there are no parallel links in VANETs, if $S R O$ is not unique, assume the other $S R O_{2}=$ $S y_{i 1} y_{i 2} \ldots y_{i k-1} T$, there is minimum one the vehicle that its vicinity in $S R O_{2}$ travel along considerable to in $S R O$, assume it is $y_{i}$, the father of $y_{i}$ is $y_{m}, m<i-1, y_{i}$ is neighbor to $y_{m}$ in $V_{T}$, then $S y_{1} y_{2} \ldots y_{m} y_{i} y_{i+1} \ldots w_{k-1} T$ is a route $S R O(S, T)$. Its distance is $<k-1$, it's conflicted to $S R O$ is a simple route, so $S R O$ is a Source-to-Terminal simple route, it is the one which is notified by its vehicles set $K_{S R O}$.

Theorem3. All the $S R O$ routes in $V_{T}$ are contained in a tree $T_{r}$, it's an SRORT tree if and only if the vehicle in an optional route is not its ancestor or brother of its ancestor.

Prove: A circle in the tree will appear if a vehicle is its ancestor, the definition of the tree is conflicting here. When a vehicle is its ancestor's brother, as shown in Fig. $2 \mathrm{~S} \rightarrow \mathrm{v} \rightarrow \mathrm{y} \rightarrow \mathrm{T}$ is a $R O(S, T)$ route, its range is lesser than the range of $\mathrm{S} \rightarrow \mathrm{v} \rightarrow \mathrm{b}(\mathrm{y}) \rightarrow \mathrm{y} \rightarrow \mathrm{T}$, the definition of the $S R O(S, T)$ route is conflicting.

If the contained route in the tree is not $S R O(S, T)$, based on the definition of $S R O(S, T)$ route, there is vehicle in the route that is it's ancestor's neighbor in $V_{T}$, then it is, it's ancestor's brother within the tree, this condition encounter with theorem. But the theorem is perfect. 


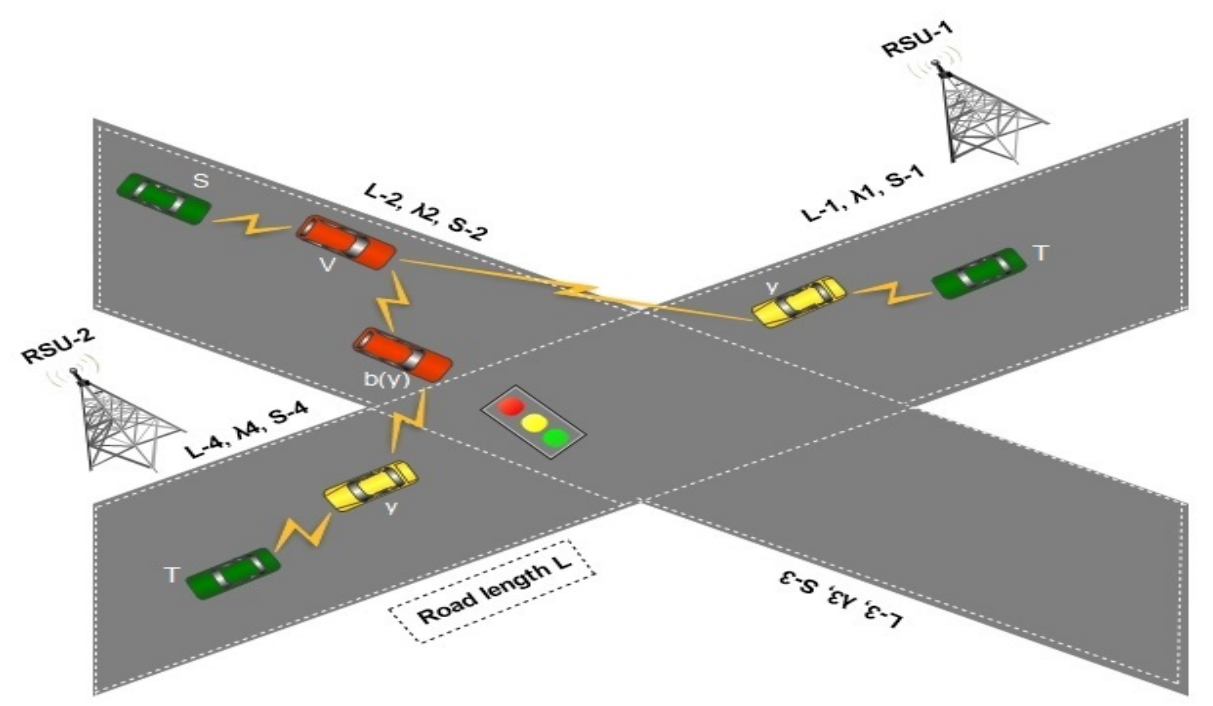

Fig. 2. A SRORT tree

Theorem 4. The reliability of $V_{T}, R\left(V_{T}\right)=\sum_{i=1}^{m} P\left(S R O_{i}\right)\left(1-R\left(V_{T_{i}}\right)\right.$, in the formula the $V_{T_{i}}$, can be made up in this way: vehicle $\mathrm{S}$ which is the Source vehicle of $V_{T}$ becomes the Source vehicle of $V_{T i}$, vehicle T which is the Terminal vehicle of $V_{T}$ becomes the Terminal vehicle of $V_{T_{i}}$, each vehicle in the middle of $S R O_{k}(k<i)$ of the $\boldsymbol{S R O R T}$ tree of $V_{T}$,

1. If Source $\mathrm{S} \rightarrow y_{j}$ route in the $S R O_{i}$ route, then the route amalgamated to the Source $\mathrm{S}$ of $V_{T_{i}}$.

2. If $y_{j}$ is the vehicle in the middle of $S R O_{k}$ route and it is a vehicle in the $S R O_{i}$ route too, in that case, remove the vehicle and link the father and the son of vehicle $y_{j}$.

3. If $y_{j} \rightarrow$ T route in the $S R O_{i}$ route, in that case, the route amalgamated to the Terminal $\mathrm{T}$ of $V_{T}$.

4. If the set of vehicles on the route is a subset of another alternative route, then eliminate the route. There is no vehicle in $V_{T_{i}}$ that it is a vehicle in the $S R O_{i}$ route except $\mathrm{S}$ and $\mathrm{T}$ vehicles.

5. The $V_{T_{i}}$ is made of these routes.

Prove: We assume SRORT contains $S r_{1}, S r_{2}, \ldots S r_{m}, b_{1}, b_{2}, \ldots b_{m}$ expressing the event functioning cooperative to the $S R O_{i}$ route.

$$
\begin{aligned}
& R\left(V_{T}\right)=P\left(b_{1}+b_{2}+\ldots+b_{m}\right) \\
& =P\left(b_{1}+\overline{b_{1}} b_{2}+\ldots+\overline{b_{1}} \overline{b_{2}} \ldots \overline{b_{i-1}} \frac{b_{i}}{1}+\ldots+\overline{b_{1}} \overline{b_{2}} \ldots \overline{b_{m-1}} b_{m}\right) \\
& \left.=P\left(b_{1}\right)+P\left(\overline{b_{1}} b_{2}\right)+\ldots+P\left(\overline{b_{1}}\right) \ldots \overline{b_{2}} \ldots \overline{b_{i-1}} b_{i}\right)+\ldots+P\left(\overline{b_{1}} \overline{b_{2}} \ldots \overline{b_{m-1}} b_{m}\right) \\
& =\operatorname{Pr}\left(b_{1}\right)+P\left(\overline{b_{1}} b_{2}\right)+\ldots+P\left(\left(\overline{b_{1}} b_{i}\right)\left(\overline{b_{2}} b_{i}\right) \ldots\left(\overline{b_{i-1}} b_{i}\right)\right) \\
& +\ldots+P\left(\left(\overline{b_{1}} b_{m}\right)\left(\overline{b_{2}} b_{m}\right) \ldots\left(\overline{b_{m-1}} b_{m}\right)\right) \\
& \quad \quad \text { we assume } S R O_{i}=c_{1}, c_{2}, c_{3}, c_{4}, c_{5}, S R O_{j}=c_{1} c_{6} c_{3} c_{7} c_{5} \text { (refer to Fig. 3) }
\end{aligned}
$$




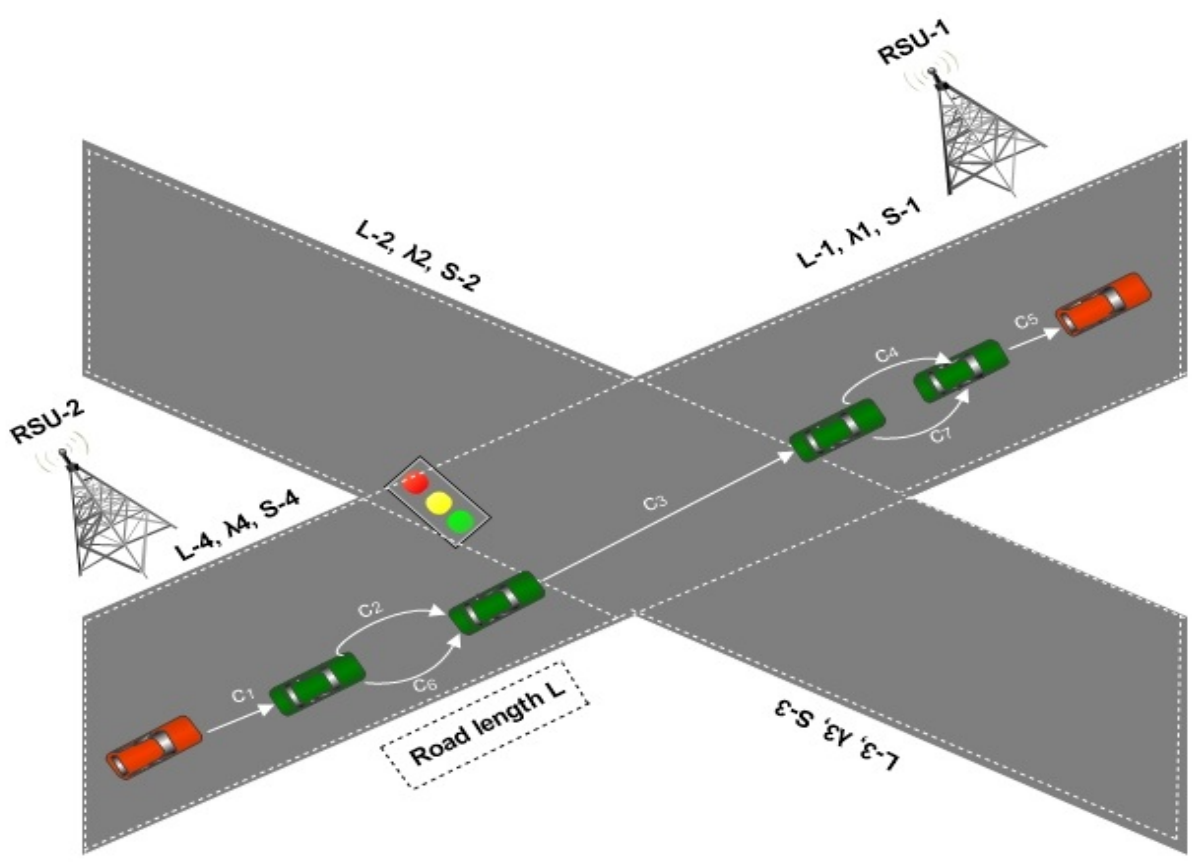

Fig. 3. Two SRO routes in the SRORT tree

$$
\begin{aligned}
& \left.\overline{b_{i}} b_{j}=\overline{c_{1} c_{2} c_{3} c_{4} c_{5}} \cap\left(c_{1} c_{6} c_{3} c_{7} c_{5}\right)\right) \\
& =\left(\overline{c_{1}}+\overline{c_{2}}+\overline{c_{3}}+\overline{c_{4}}+\overline{c_{5}}\right) \cap\left(c_{1} c_{6} c_{3} c_{7} c_{5}\right) \\
& =\overline{c_{2}}\left(c_{1} c_{6} c_{3} c_{7} c_{5}\right)+\overline{c_{4}}\left(c_{1} c_{6} c_{3} c_{7} c_{5}\right) \\
& =\overline{c_{2} c_{3}}\left(c_{1} c_{6} c_{3} c_{7} c_{5}\right) \\
& =\overline{c_{2} c_{3}} b_{j}
\end{aligned}
$$

This $c_{2} c_{4}$ is a $S R O(S, T)$ route of $V_{T_{i}}$, then

$$
\begin{aligned}
& R\left(V_{T}\right)=P\left(b_{1}\right)+P\left(\overline{V_{T_{1}}} b_{2}\right)+\ldots+P\left(\overline{V_{T_{i}}} b_{i}\right)+\ldots+P\left(\overline{V_{T_{m}}} b_{m}\right) \\
& \quad=P\left(b_{1}\right)+\left(1-P\left(V_{T_{1}}\right)\right) P\left(b_{2}\right)+\ldots \\
& \quad+\left(1-P\left(V_{T_{i}}\right)\right) P\left(b_{i}\right)+\ldots+\left(1-P\left(V_{T_{m}}\right)\right) P\left(b_{m}\right)
\end{aligned}
$$

\section{Algorithm: ${ }^{[7]}$}

$S$ : initialization state Source vehicle;

OPEN table: the table saving vehicle that not extended;

CLOSED table: the table saving vehicle that extended;

1. Input probabilistic network $\left(V_{T}\right)$ and the Source \& Terminal vehicles $(S, T)$, $R(\Phi)=0, i=1$

2. Generate a search network only including $\mathrm{S}$, put $\mathrm{S}$ into the OPEN table;

3. Generate a CLOSED table and insert it as an empty table; 
4. When the OPEN table is an empty table, then output $R\left(V_{T}\right)$, exit ;

5. Choose the first vehicle in the OPEN table, take it out from the OPEN table and insert it into the CLOSED table, mark that vehicle as v;

6. If the vehicle $\mathrm{v}$ is the Terminal vehicle then search a simple route $(S R O(S, T))$, if each $S R O(S, T)$ in $V_{T_{1}}$ have not the same vehicle as compared to other $S R O(S, T)$ in $V_{T_{1}}$, then

$$
R\left(V_{T}\right)=R\left(V_{T}\right)+\left(1-\prod \text { all SRO in } V_{T_{1}} P\left(\operatorname{SRO}\left(V_{T_{1}}\right)_{k}\right)\right) P\left(S R O_{i}\right),
$$

else, compute recursively $R\left(V_{T_{1}}\right)$ according to theorem 4 ,

$$
R\left(V_{T}\right)=R\left(V_{T}\right)+\left(1-R\left(V_{T_{1}}\right)\right) P\left(S R O_{i}\right) ; i=i+1 \text {, proceed to } 4 ;
$$

7. Extend the vehicle $v$, generate a vehicles set and those vehicles are not its ancestor and brother of its ancestor, mark the set as $\mathrm{S}(\mathrm{v})$, when $\mathrm{S}(\mathrm{v})=\Phi$, after that from CLOSED table remove the vehicle $\mathrm{v}$, proceed to (4), else put the vehicles in $\mathrm{S}(\mathrm{v})$ into the search $V_{T}$ as succession vehicle v;

8. The vehicles in $\mathrm{S}(\mathrm{v})$, set an indicator to its father vehicle $\mathrm{v}$ and in the OPEN table it is inserted;

9. According to the DFS policy arrange the vehicles in the OPEN table, proceed to 4;

The algorithm is explained in Fig. 4.

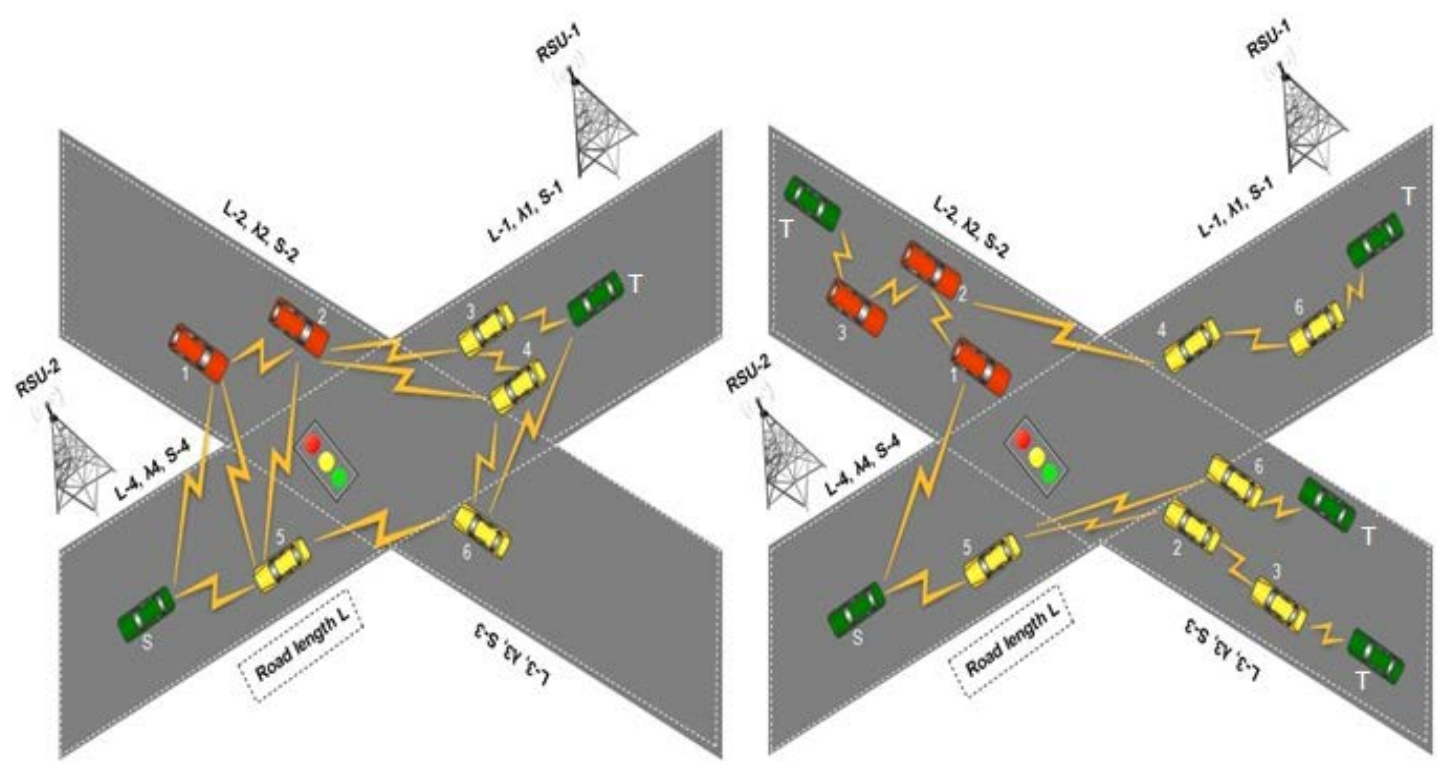

Fig. 4. $\left(V_{T}\right)$ VANET and its SRORT tree with universal speed factor model.

This algorithm is recursive, in the first time cycle, find a simple route $\left(S R O_{1}\right), V_{T_{1}}=$ $\Phi, R\left(V_{T_{1}}\right)=0$. In the second time cycle, $V_{T_{3}}=V_{3}$

$R\left(V_{T}\right)=P\left(V_{1} V_{2} V_{3}\right)+\left(1-R\left(V_{T_{2}}\right)\right) P\left(V_{1} V_{2} V_{4} V_{6}\right)=P_{1} P_{2} P_{3}+\overline{P_{3}} P_{1} P_{2} P_{4} P_{6}$

Keep on searching in this way, generate $S_{R} O_{3}, S R O_{4}$, obtained $V_{T_{3}}, V_{T_{4}}$

$$
V_{T_{3}}=x_{1}
$$




$$
\begin{aligned}
R\left(V_{T}\right)=R\left(V_{T}\right)+ & \left(1-R\left(V_{T 3}\right)\right) P\left(V_{5} V_{2} V_{3}\right) \\
& =R\left(V_{T}\right)+\overline{P_{3}} P_{2} P_{3} P_{5}
\end{aligned}
$$

$V_{T_{4}}$ have two simple routes and they have the same vehicle, we compute recursively

$$
\begin{gathered}
V_{T_{4}}=V_{2} V_{3}+V_{1} V_{2} V_{4} \\
R\left(V_{T}\right)=R\left(V_{T}\right)+\left(1-R\left(V_{T 4}\right)\right) P\left(V_{5} V_{6}\right) \\
\left.=R\left(V_{T}\right)+\overline{P_{2}\left(P_{3}+\overline{P_{3}} P_{1}\right)} P_{5} P_{6}\right)
\end{gathered}
$$

The given expression is obtained when the OPEN table is the empty table,

$$
\begin{aligned}
R\left(V_{T}\right)=P & \left(V_{1} V_{2} V_{3}+\overline{V_{3}} V_{1} V_{2} V_{4} V_{6}+\overline{V_{1}} V_{2} V_{5} V_{6}+\overline{V_{2}\left(V_{3}+\overline{V_{3}} V_{1}\right)} V_{5} V_{6}\right) \\
& \left.=P_{1} P_{2} P_{3}+\overline{P_{3}} P_{1} P_{2} P_{4} P_{6}+\overline{P_{1}} P_{2} P_{5} P_{6}+\overline{P_{2}\left(P_{3}+\overline{P_{3}} P_{1}\right.}\right) P_{5} P_{6}
\end{aligned}
$$

\section{Universal Speed Factor (USF)}

The same distribution as that of the queuing system model in [23] is followed in this paper. Assume a one-directional road of length $L$ contains sub-segment $S=\left\{s_{1}, s_{2} \ldots s_{n}\right\}$ refer to Fig. 1. At the highway, the arrival of vehicles follows Poisson distribution along mean $\frac{1}{\lambda}$ per unit time. The arrival rate is constant $\lambda_{i}(i=1,2,3 \ldots n)$ with unit veh/h for each road segment $s_{i}$. Let suppose that $\lambda_{1}=\lambda_{2}$ and $\lambda_{3}=\lambda_{4}$. The headway distance and inter-arrival time of two vehicles follow the exponential distribution. The vehicles cannot travel freely in Fig. 1, because of the intersection. These vehicles on segment $S-1, S-2$ and $S-3$ will follow the $S-4$ direction after crossing the intersection. Refer to Fig. 1.

We assume each vehicle with different velocity entering the road segment $s$. The steady-state distribution of vehicles is considered by the authors of [14] [22] and [23]. In their models, they assumed constant speed for a specified time duration and they modeled speed as Gaussian distribution. When assigning $v$ as a random speed to a vehicle, then probability density function of Gaussian distribution is [13] [15] [24].

$$
f(v)=\frac{1}{\sigma \cdot \sqrt{2 \pi}} e^{-\frac{(v-u)^{2}}{2 \sigma^{2}}}
$$

The mean and standard deviation of speed $v$ is represented by $\mu$ and $\sigma$. A new parameter named speed factor $A$ was introduced by the authors in [14]. The intensity of vehicles on the highway is shown with parameter $A$ with estimating unit $\mathrm{h} / \mathrm{Km}$ and reflects the effect of speed on headway distance as shown below analytically.

$$
A=\int_{v_{\min }}^{v_{\max }} \frac{\hat{f_{v}}(v)}{v} d(v)
$$




$$
\hat{f}_{v}(v)=\frac{f_{v}(v)}{\int_{v_{\min }}^{v_{\max }} f_{v}(S) d(S)}
$$

where upper and lower speed limits of vehicles are $v_{\max }$ and $v_{\min }$. Limitation: The same speed is considered for all the vehicles including same mean $\mu$ and $\sigma$ standard deviation, which follows the Gaussian distribution. Considering same speed at all-time seriously affects the headway distance on highways. The headway distance between vehicles will become steady as long as speed remains consistent and the situation will be unrealistic.

We revised the speed factor $A$ with USF, which depicts the vehicles intensity on a sub-segment of road $s$ with evaluating unit $\mathrm{h} / \mathrm{Km}$ and reflects the effects of relative speed on headway distance. The proposed USF metric is based on the relative speed with distinctive mean $\mu$ and $\sigma$ standard deviation, as given.

$$
\begin{gathered}
V_{1} \sim n\left(\mu_{1}, \sigma_{1}^{2}\right) \\
V_{2} \sim n\left(\mu_{2}, \sigma_{2}^{2}\right) \\
V_{3} \sim n\left(\mu_{3}, \sigma_{3}^{2}\right) \\
\vdots \\
\vdots \\
V_{n-1} \sim n\left(\mu_{n-1}, \sigma_{n-1}^{2}\right) \\
V_{n} \sim n\left(\mu_{n}, \sigma_{n}^{2}\right)
\end{gathered}
$$

The vehicles on the highway are $\left\{V_{1}, V_{2} \ldots V_{n-1}, V_{n}\right\}$. With different mean and variance, each vehicle follows the Normal distribution, because of the road condition and drivers intention. The relative velocities and probability density functions will be $f\left(v_{12}\right) \sim n\left(\mu_{12}, \sigma_{12}^{2}\right)$, where $v_{12}=v_{1}-v_{2}, \mu_{12}=\mu_{1}-\mu_{2}$ and $\sigma_{12}^{2}=\sigma_{1}^{2}+\sigma_{2}^{2}$. If the two vehicles $i$ and $j$ having differential velocity $v$, then

$$
f\left(v_{r e l}\right)=\frac{1}{\sqrt{\sigma_{i}^{2}+\sigma_{j}^{2} \cdot \sqrt{2 \pi}}} e-\frac{(v-(v i-v j))^{2}}{2\left(\sigma_{i}^{2}+\sigma_{j}^{2}\right)}
$$

The deviations of vehicle $i$ and $j$ are $\sigma_{i}$ and $\sigma_{j}$. The probability density function $f\left(v_{\text {rel }}\right)$ denotes the normal distribution of speed. According to (12), the description of USF becomes.

$$
\begin{gathered}
U S F=\int_{v_{\min }}^{v_{\max }} \frac{f_{v_{\text {rel }}}^{*}(v)}{v} d(v) \\
f_{v}^{*}\left(v_{r e l}\right)=\frac{f_{v}\left(v_{r e l}\right)}{\int_{v_{\min }}^{v_{\max }}(s) d(s)}
\end{gathered}
$$

The truncated velocities are $v_{\min }$ and $v_{\max }$. USF uses exponential distribution based on variable arrival rate [13] [15] as shown in (15)

$$
f\left(\frac{s}{\mu}\right)=\frac{1}{\mu} e^{\frac{-s}{\mu}}
$$

For a given mean $\mu$ (15) gives the probability of $s$ unit headway distance. 


\section{Connectivity of VANETs for Different Mobility Environments}

The connectivity probability $p_{c}$ of the vehicles is the main concern of this paper using USF in high-speed scenarios. As connectivity is a function of vehicle density, hence in both mobile and static scenarios, spatial density should be evaluated.

\subsection{Evaluation of Vehicle Density}

The spatial density of vehicles defines the vehicles intensity on road [20] [15]. The node spatial density has an opposite relation with road length $L$. Assume $s$ a road segment having length $L_{s}$ including vehicles arrival rate $\lambda$ from $t_{0}$ to $t_{1}$. Then on road segment $s$ vehicle, spatial density $\rho$ is

$$
\rho=\frac{\left(t_{1}-t_{0}\right) \lambda}{L_{s}}
$$

Where in the time interval $\left[t_{0}-t_{1}\right] \rho$ is the spatial density of vehicle. In the mobility case, assume with $\lambda$ arrival rate of vehicles at road segment $s$ with average relative speed factor USF, then the number of average vehicles $n$ at time $t$ is

$$
n=L_{S} \lambda \cdot(U S F)
$$

In (17) USF is the average speed factor as derived in (13). Along with the proposed connectivity definition, the proof of (17) is shown in Appendix.

\subsection{Static Connectivity}

In the static environment, on the road segment $s$ vehicles will make a stationary platoon. Suppose at time $t$, a sorted queue is formed by vehicles based on the location indicated as $\left\{V_{1}, V_{2}, V_{3} \ldots V_{n}\right\}$ on road segment of length $L$. Suppose the random variable $X_{i}$ represents the headway distance between vehicles $V_{i}$ and $V_{i+1}$ [13] [15]. If there is, a route connecting any two vehicles the VANET is connected. It is very clear now that the distance amongst any two vehicles must be lesser than the transmission range of the vehicles $R$. Let in a sub segment $s$ the connectivity probability $P_{c}$ of vehicles [13] [15], then

$$
P_{c}=\operatorname{pr}\left\{X_{1}<R, X_{2}<R \ldots n d_{n-1}<R\right\}
$$

where $X_{i}$ is a $i, i, d$ random variable and $R$ is the vehicles transmission range. When at least $k$ vehicles are connected on road segment $s$ the connectivity probability $P_{c}$ is

$$
P_{c}(k)=P\left(n \geq k=\sum_{i=k}^{\infty} P(n=i)=\sum_{i=k}^{\infty}\left(F_{S}(R)\right)^{i}=\left(1-e^{-\rho \mathrm{R}}\right)^{k-1}\right.
$$

In (19), $\boldsymbol{F}_{\boldsymbol{s}}($.$) is the distribution function of the headway distance. k$ shows the connected vehicles at time $t$ and $n$ is the number of total vehicles, $\boldsymbol{\rho}$ is defined in (16). The node mobility is neglected in (19). Once introduce the mobility, the increase in connectivity will be observed, this is the main contribution and motivation of this paper. 


\subsection{The case of mobile connectivity}

The core parameter which affects the VANET performance in all aspects is mobility. Because of the lack of deep analysis of real-time vehicular mobility traces, we are unable to recognize the connectivity in real-time scenarios. Specially, the method of how the mobility of networked vehicles effects the network connectivity remains unidentified. The main concern of this paper is free flow connectivity [25]. The probability of having $k$ vehicles connected in a road segment $s$ with transmission range $R$ and arrival rate $\lambda_{i}$ is

$$
P_{c}(k)=P\left(n \geq k=\sum_{i=k}^{\infty}\left(1-e^{\left.-L_{S} \lambda_{i}(U S F) \frac{R}{L}\right)}\right.\right.
$$

The number of total vehicles is $n$ and USF is the proposed USF given in (13). In the Appendix, proof of (20) is given. By using (17), it gives

$$
P_{c}(k)=P\left(n \geq k=\sum_{i=k}^{\infty}\left(1-e^{\left.-n_{s} \frac{R}{L}\right)}\right.\right.
$$

where the average number of vehicles is $n_{s}, L$ is the highway length and the fixed transmission range of every vehicle is $R$.

\section{Discussions and Obtained Results}

This section compares the performance of the USF with the existing method [14] in terms of connectivity and tested its efficiency and likelihood in different simulation scenarios.

We divide this section into two subsections. In the first section, the congested and static scenario including its comparison with the existing method is highlighted. The second section represents the simulation performance of USF in high mobility scenario.

\subsection{The case of static and slow mobility analysis}

The static scenario as shown in Fig. 1, examines the scenario on intersections, where vehicles are considered static. Since the vehicles are static, the proposed USF is not considered and the relation between connectivity $P_{c}$ and node spatial density is analyzed by (19).

With the arrival rate of $\lambda=\{0.1,0.5,1\}$ veh/sec, the temporal-spatial density $\rho$ is considered as given (16). The relation between connectivity and temporal-spatial density is depicted in Fig. 5. 


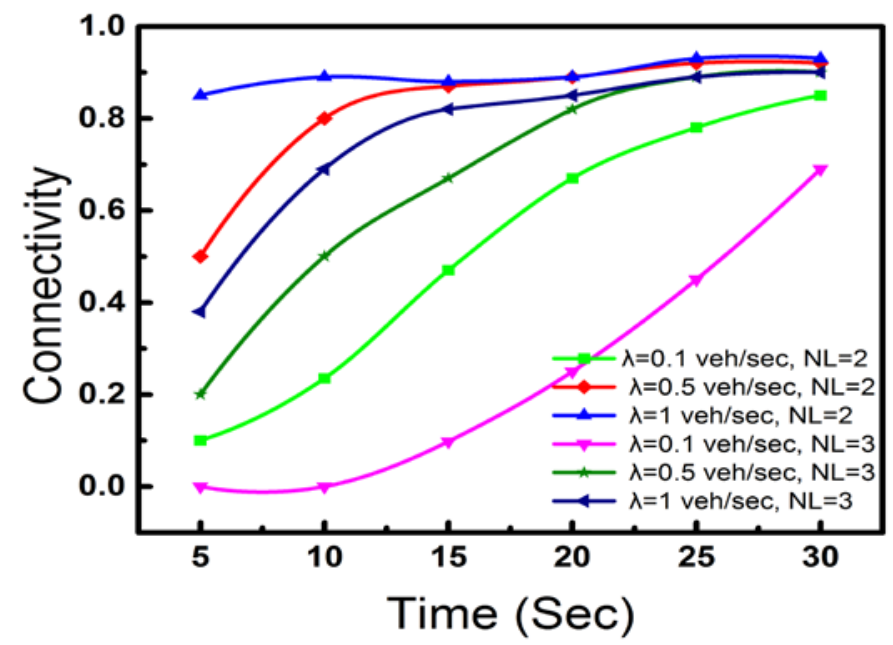

Fig. 5. Connectivity versus spatial node density (static scenario, length of road $L=10 \mathrm{~km}$, number of road lanes $\mathrm{NL}$ ).

It is shown in the results that under different arrival rates $(\lambda=0.1,0.5,1 \mathrm{veh} / \mathrm{sec})$, with the increase in arrival rate connectivity increases. The connectivity of vehicles also affected by road lanes. It is observed that by keeping the same arrival rate as compared to three lanes road the connectivity of two lanes road will be higher as shown in Fig. 5.

Now we consider the slow mobility with mean velocity $\mu 20 \mathrm{Km} / \mathrm{h}$ and $40 \mathrm{Km} / \mathrm{h}$. The USFs of $20 \mathrm{Km} / \mathrm{h}$ and $40 \mathrm{Km} / \mathrm{h}$ are $0.07271 \mathrm{~h} / \mathrm{Km}$ and $0.07096 \mathrm{~h} / \mathrm{Km}$ respectively using (13) with $\mu_{d}=21 \mathrm{~m}$ and $\sigma=21 \mathrm{Km} / \mathrm{h}$.

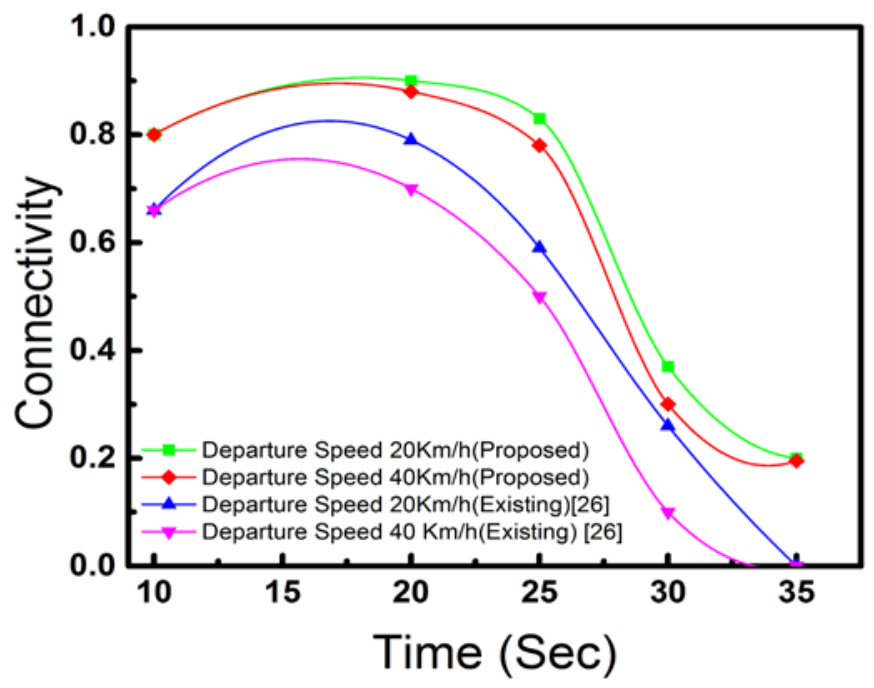

Fig. 6. The impact of spatial node density and slow mobility on connectivity $(\mathrm{R}=500 \mathrm{~m}$, road length $\mathrm{L}$ $=1 \mathrm{Km}$ ). 
The connectivity is affected by mobility as shown in Fig. 6. The connectivity probability is improved by $20 \%$ with the proposed USF as suggested by the results. In the case of slow mobility scenario, the proposed USF outperform than existing method [14] as shown in the line graph.

As a conclusion, we observe that using exponential distribution as headway distance and relative speed can improve the overall vehicle's connectivity.

\subsection{The case of high mobility analysis}

In the mobility scenario, connectivity is a function of USF as defined in (13) including transmission range $R$ and arrival rate. In the high-speed scenarios, it is shown that the connectivity probability $P_{c}$ is affected by mobility positively up-to $\mu_{\tau}$ and once the mean velocity exceeds $\mu_{\tau}$, the decrease in the connectivity is observed as shown in Fig. 7, 8. With vehicle density 100 and 200 the $\mu_{\tau}$ in Fig. 7 is 80 and $85 \mathrm{Km} / \mathrm{h}$ respectively. The influence of speed factor and transmission range $R$ on connectivity is also given in Fig. 8. The $\mu_{\tau}$ becomes 80 and $90 \mathrm{Km} / \mathrm{h}$, in the case of transmission range $R$ as shown in Fig. 8. As compared to existing methods simulation shows better connectivity by adding USF as shown in Fig. 7, 8.

Both slow and high mobility scenarios are analyzed in the simulation section. Based on the results, it is observed that in slow mobility scenarios, the connectivity decreases slowly after passing the threshold limit.

But in the case of high mobility scenarios, after passing $\mu_{\tau}$ connectivity decreases very fast.

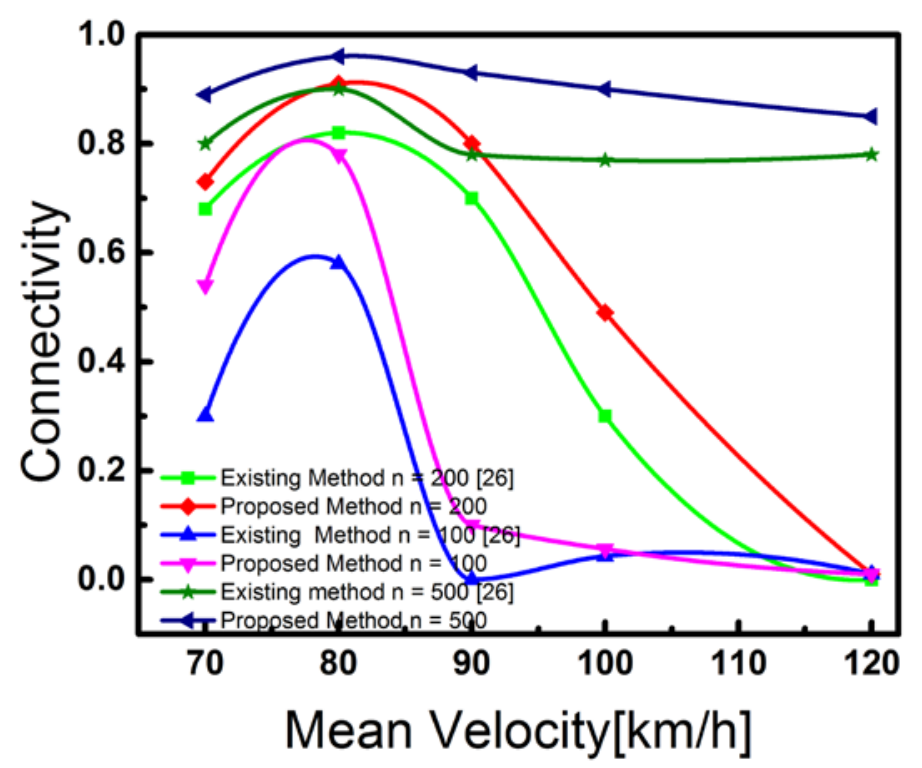

Fig. 7. Proposed versus existing connectivity method $\left(\sigma=40 \mathrm{Km} / \mathrm{h}\right.$, exponential mean $\mu_{d}=100 \mathrm{~m}, \mathrm{R}$ $=500 \mathrm{~m}$, road length $=5 \mathrm{Km}, n$ : number of vehicles). 


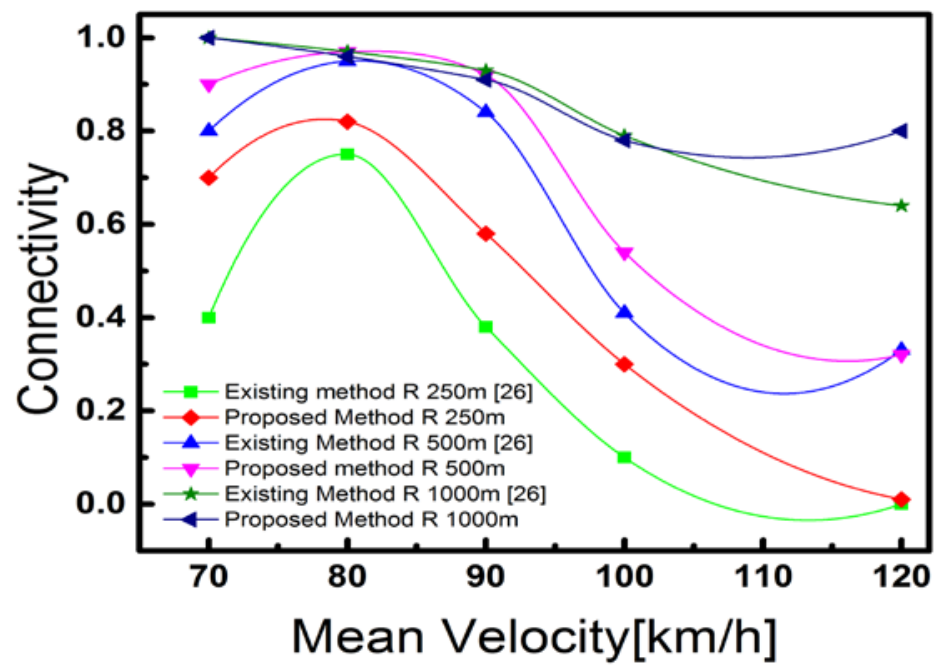

Fig. 8. Proposed versus existing connectivity method (exponential mean $\mu_{d}=100 \mathrm{~m}, \sigma=40 \mathrm{Km} / \mathrm{h}, \mathrm{R}$ $=500 \mathrm{~m}$, road length $=5 \mathrm{Km}, n$ : number of vehicles $=500)$.

\section{Conclusions}

The number of simple routes (SRO) is less than the number of Source-to-Terminal routes, $V_{T_{i}}$ is a simple network, $R\left(V_{T_{i}}\right)$ can be easily computed. The workload at 1 st hand apply Inclusion-Exclusion principle or enumerate Source-to-Terminal route is very long, that's why the expression for $R\left(V_{T}\right)$ is long. Compare our algorithm the workload is less, also the expression is short. This is the advantage of our algorithm. The enhanced mobility factor USF offers an improved stochastic connectivity of vehicles. The connectivity is a function of node density, transmission range, and mobility. All the parameters are directly related to the connectivity. An extremely congested traffic environment will be extremely connected. The connectivity will be increasing until a given threshold but will decrease after reaching that threshold.

\section{Conflicts of Interest}

"I declare that none of the authors have a conflict of interest."

\section{ACKNOWLEDGMENT}

This work is supported by the National Natural Science Foundation of China (61370201), Open Research Found from the Key Laboratory for Computer Network and Information Integration (Southeast University, Ministry of Education, China). 


\section{Appendix}

Proof of the mobile case connectivity EQ. (20)

Assume $n$ vehicles with radio propagation range $R$ are traveling on a road segment $s$ of length $L$ with the vehicles arrival rate $\lambda$ from time $t_{0}$ to $t_{1}$. Suppose that the condition of the road is normal, then only two depended parameters are left such as speed and arrival rate.

Case I: Time-Variant Arrival rate and connectivity: The number of vehicles crossing through road segment $s$ in the time interval $\left[t_{i} t_{j}\right]$ with time variant intensity rate $\lambda$ is computed as below.

$$
V=\sum_{k=i}^{j}\left(T_{k} \lambda_{k}\right)
$$

Consider the departure rate $\lambda^{*}$ (fixed at every interval of time), then spatial density $\rho$ will be

$$
\rho=\frac{\sum_{k=i}^{j}\left(T_{k} \lambda_{k}-\lambda^{*}\right)}{L}
$$

Hence the description of connectivity will change to the time-variant arrival of vehicles by putting (23) in (20).

Case II: Vehicle Speed and Connectivity: Assume speed of all the vehicles follow Gaussian distribution $n\left(\mu, \sigma^{2}\right)$ having unique mean $\mu$ and standard deviation $\sigma$. In literature [12], the authors presented a parameter which shows the inter-distances and speed association by $\frac{\lambda}{\mu}$. To find the number of vehicle, it is known that, number of vehicles $=$ highway length $\times$ density of vehicles. Hence

$$
n=\frac{L_{s} \lambda}{\mu}
$$

In the proposed case, the USF is considered as given in (13) instead of fixed speed and vehicle arrival rate. Since USF parameter is an inverse of normal speed, which correlates the inter-vehicle spacing with relative speed.

$$
\begin{array}{r}
\text { Veh Density }(d)=(U S F) \cdot \lambda \\
\text { number of vehicles }(n)=(U S F) \lambda L_{S}
\end{array}
$$

Spatial density $\rho$ can be calculated as below

$$
\rho=\frac{(U S F) \lambda L_{S}}{L}
$$

USF is the proposed speed factor. Hence by putting (26) in (20), the new definition based on vehicle mobility is obtained. 


\section{References}

[1] Satyanarayana, A., and A. Prabhakar, "New topological formula and rapid algorithm for reliability analysis of complex networks," IEEE Transactions on Reliability vol.27(2), pp.82-100, 1978. Article (CrossRef Link).

[2] Zhao, L. C., \& Kong, F. J., "A new formula and an algorithm for reliability analysis of networks,” Microelectronics Reliability, vol.37(3), pp.511-518, 1997. Article (CrossRef Link).

[3] Locks, M. O., "Recent developments in computing of system-reliability," IEEE transactions on reliability, vol.34(5), pp.425-436, 1985. Article (CrossRef Link).

[4] Gao, F., Zhao, C., Hu, H., \& Yang, J., “An algorithm reliability of wireless networks for sum of disjoint product and topological structure," WSEAS TRANSACTIONS on COMMUNICATIONS, vol.8(1), pp.61-70, 2009. Article (CrossRef Link).

[5] Aboeifotoh, H. M., \& Colbourn, C. J., "Efficient algorithms for computing the reliability of permutation and interval graphs,” Networks, vol.20(7), pp.883-898, 1990. Article (CrossRef Link).

[6] Krivelevich, M., Sudakov, B., \& Vu, V. H, “A sharp threshold for network reliability," Combinatorics, Probability and Computing, vol.11(5), pp.465-474, 2002. Article (CrossRef Link).

[7] Gao, F., Liu, X., \& Liu, H., "A rapid algorithm for computing ST reliability of radio-communication networks," in Proc. of In International Conference on Knowledge-Based and Intelligent Information and Engineering Systems, pp. 167-174, 2005. Article (CrossRef Link).

[8] S. Singh, N. Saxena, A. Roy, and H. Kim, “A survey on 5G network technologies from social perspective,” IETE Tech. Rev., vol. 34, no.1, pp. 30-39, 2017. Article (CrossRef Link).

[9] K. Zheng, Q. Zheng, P. Chatzimisios, W. Xiang, and Y. Zhou, "Heterogeneous vehicular networking: A survey on architecture, challenges, and solutions,” IEEE Commun. Surveys Tuts., vol. 17, no. 4, pp. 2377-2396,4th Quart., 2015. Article (CrossRef Link).

[10] N. Lu, N. Cheng, N. Zhang, X. Shen, and J. W. Mark, "Connected vehicles: Solutions and challenges,” IEEE Internet Things J., vol. 1, no. 4,pp. 289-299, Aug. 2014.

Article (CrossRef Link).

[11] Z. Zhang, G. Mao, and B. D. O. Anderson, “On the information propagation process in mobile vehicular ad hoc networks,” IEEE Trans. Veh. Technol., vol. 60, no. 5, pp. 2314-2325, Jun. 2011. Article (CrossRef Link).

[12] S. Durrani, X. Zhou, and A. Chandra, "Effect of vehicle mobility on connectivity of vehicular ad hoc networks," in Proc. IEEE 72nd Veh. Technol. Conf. Fall (VTC-Fall), pp. 1-5, Sep. 2010. Article (CrossRef Link).

[13] S. Panichpapiboon and W. Pattara-atikom, "Connectivity requirements for self-organizing traffic information systems,” IEEE Trans. Veh. Technol.,vol. 57, no. 6, pp. 3333-3340, Nov. 2008. Article (CrossRef Link).

[14] C. Chen, X. Du, Q. Pei, and Y. Jin, "Connectivity analysis for free-flow traffic in VANETs: A statistical approach,” Int. J. Distrib. Sensor Netw., vol. 9(10), Aug. 2013. Article (CrossRef Link).

[15] C. Yab Shao, S. Leng, Y. Zhang, A. Vinel, and M. Jonsson, “Analysis of connectivity probability in platoon-based vehicular ad hoc networks," in Proc. of Int. Wireless Commun. Mobile Comput. Conf. (IWCMC), pp. 706-711, Aug. 2014. Article (CrossRef Link).

[16] E. Lee, E.-K. Lee, M. Gerla, and S. Y. Oh, "Vehicular cloud networking: Architecture and design principles,” IEEE Commun. Mag., vol. 52, no. 2, pp. 148-155, Feb. 2014. Article (CrossRef Link).

[17] E. Baccelli, P. Jacquet, B. Mans, and G. Rodolakis, "Highway vehiculardelay tolerant networks: Information propagation speed properties,” IEEE Trans. Inf. Theory, vol. 58, no. 3, pp. 1743-1756, Mar. 2012. Article (CrossRef Link). 
[18] R. Nagel, "The effect of vehicular distance distributions and mobility on VANET communications,” in Proc. of IEEE Intell. Vehicles Symp. (IV), pp. 1190-1194, Jun. 2010. Article (CrossRef Link).

[19] A. Cardote, S. Sargento, and P. Steenkiste, “On the connection availability between relay nodes in a VANET,” in Proc. of IEEE Globecom Workshops, pp. 181-185, Dec. 2010. Article (CrossRef Link).

[20] C. Shao, S. Leng, Y. Zhang, A. Vinel, and M. Jonsson, "Performance analysis of connectivity probability and connectivity-aware MAC protocol design for platoon-based VANETs,” IEEE Trans. Veh. Technol., vol. 64, no. 12, pp. 5596-5609, Dec. 2015. Article (CrossRef Link).

[21] Dharmaraja, S., Vinayak, R., \& Trivedi, K. S, "Reliability and survivability of vehicular ad hoc networks: An analytical approach,” Reliability Engineering \& System Safety, vol.153, 28-38, 2016. Article (CrossRef Link).

[22] A. Babu and V. K. M. Ajeer, "Analytical model for connectivity of vehicular ad hoc networks in the presence of channel randomness,” Int.J. Commun. Syst., vol. 26, no. 7, pp. 927-946, 2013. Article (CrossRef Link).

[23] N. P. Chandrasekharamenon and B. AnchareV, "Connectivity analysis of one-dimensional vehicular ad hoc networks in fading channels,” EURASIP J. Wireless Commun. Netw., vol. 2012, pp. 1-6, Dec. 2012. Article (CrossRef Link).

[24] M. J. Khabbaz, W. F. Fawaz, and C. M. Assi, “A simple free-flow traffic model for vehicular intermittently connected networks,” IEEE Trans. Intell. Transp. Syst., vol. 13, no. 3, pp. 1312-1326, Sep. 2012. Article (CrossRef Link).

[25] X. Hou, Y. Li, D. Jin, D. O. Wu, and S. Chen, "Modeling the impact of mobility on the connectivity of vehicular networks in large-scale urban environments," IEEE Trans. Veh. Technol., vol. 65, no. 4, pp. 2753-2758, Apr. 2016. Article (CrossRef Link).

[26] Z. Zhang, G. Mao, and B. D. O. Anderson, "Stochastic characterizationof information propagation process in vehicular ad hoc networks,” IEEETrans. Intell. Transp. Syst., vol. 15, no. 1, pp. 122-135, Feb. 2014. Article (CrossRef Link).

[27] Kong, X., Xia, F., Wang, J., Rahim, A., \& Das, S. K, “Time-location-relationship combined service recommendation based on taxi trajectory data," IEEE Transactions on Industrial Informatics, vol.13(3), pp.1202-1212, 2017. Article (CrossRef Link).

[28] Tao, J., Xu, Y., Zhang, Z., Feng, F., Tong, F., \& Dong, F, “A resource allocation game with restriction mechanism in VANET cloud,” Concurrency and Computation: Practice and Experience, vol.29(14), 2017. Article (CrossRef Link).

[29] Zheng, J., \& Wang, Y., "Connectivity Analysis of Vehicles Moving on a Highway with an Entry and Exit," IEEE Transactions on Vehicular Technology, vol.67(5), pp.4476-4486, 2018. Article (CrossRef Link).

[30] Venkatramana, D. K. N., Srikantaiah, S. B., \& Moodabidri, J., "CISRP: connectivity-aware intersection-based shortest path routing protocol for VANETs in urban environments,” IET Networks, vol.7(3), pp.152-161, 2018. Article (CrossRef Link).

[31] Padma, G. V., Kishore, K. H., \& Sindura, S. J., "Controlling the Traffic Interactions with High Mobility and Constant Network Connectivity by Vanets," in Proc. of 2nd International Conference on Micro-Electronics, Electromagnetics and Telecommunications, pp. 593-601, 2018. Article (CrossRef Link).

[32] Aadil, F., Khan, S., Bajwa, K. B., Khan, M. F., and Ali, A., "Intelligent Clustering in Vehicular ad hoc Networks," KSII Transactions on Internet and Information Systems, vol.10, no.8, pp.3512-3528, 2016. Article (CrossRef Link).

[33] Kong X, Li M, Tang T, et al., "Shared Subway Shuttle Bus Route Planning Based on Transport Data Analytics [J],” IEEE Transactions on Automation Science \& Engineering, vol.15(4), pp.1-14, 2018. Article (CrossRef Link). 
[34] Kong X, Li M, Li J, et al., "CoPFun: an urban co-occurrence pattern mining scheme based on regional function discovery [J]," World Wide Web-internet \& Web Information Systems, pp.1-26, 2018. Article (CrossRef Link).

[35] Xia, F., Wang, J., Kong, X., Wang, Z., Li, J., \& Liu, C., "Exploring human mobility patterns in urban scenarios: a trajectory data perspective,” IEEE Communications Magazine, vol.56(3), pp.142-149, 2018. Article (CrossRef Link).

[36] Kong X, Li M, Ma K, et al., "Big Trajectory Data: A Survey of Applications and Services,” IEEE Access, vol.6, pp.58295-85306, 2018. Article (CrossRef Link).

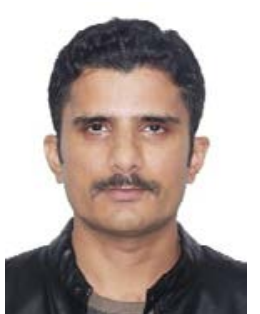

Hussain Saajid received his BSIT degree from Sindh Agriculture University, Pakistan in 2009. He received his M.S. degree in Software Application and Technology from Central South University, Hunan, Changsha, China in 2015. Now he is a Ph.D. candidate at Dalian University of Technology, Liaoning, China. His research interests include Vehicular ad hoc Networks.
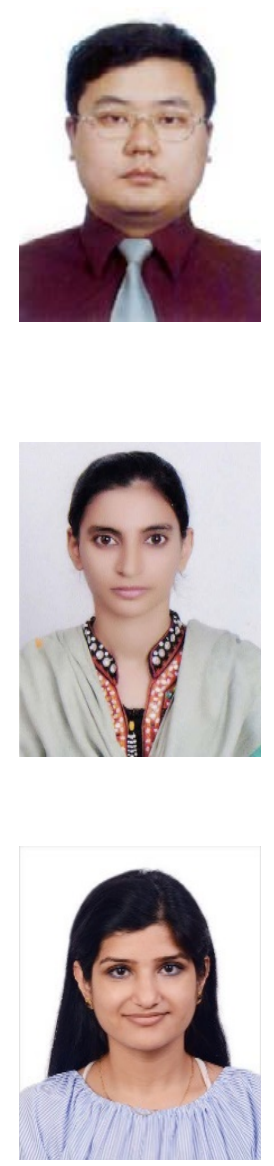

Di. Wu born in April 1972, Liaoning, associate professor and master tutor of School of Computer Science and Technology /Dalian University of Technology, Dalian, China. The main research directions are the VANETs, Internet, wireless ADHOC networks, computer network and the computer simulation application in the engineering, etc. Since 2001, he is the associate professor of Dalian university of technology, college of computer and the Internet of things institute. 2009-2010, he as a visiting scholar to the Canadian Simon Fraser University, college of compute.1999-2001, he is the postdoctoral in Northeastern university, institute of computer application and the network and communication center. He has published more than 50 papers and 2 patents in domestic and international academic conferences and journals. He is a senior member of computer society.

Sheeba Memon received her BSIT degree from Mehran University of Technology, Pakistan in 2011. She received her M.S. degree in Computer Science from Isra University, Hyderabad, Pakistan in 2015. Now she is a Ph.D. candidate at Central South University, Hunan, Changsha, China. Her research interests include Data Center Networks, Vehicular ad hoc Networks.

Naadiya Khuda Bux received her BSIT degree from Sindh Agriculture University, Pakistan in 2011. She received her M.S. degree in Computer Science from Isra University, Hyderabad, Pakistan in 2014. Her area of interests include data mining, machine learning and big data. 